ARTICLE

\title{
Human serum-derived protein removes the need for coating in defined human pluripotent stem cell culture
}

Sara Pijuan-Galitó1, Christoffer Tamm¹, Jens Schuster ${ }^{2}$, Maria Sobol ${ }^{2}$, Lars Forsberg ${ }^{2}$, Catherine L.R. Merry ${ }^{1,3}$ \& Cecilia Annerén 1,4

Reliable, scalable and time-efficient culture methods are required to fully realize the clinical and industrial applications of human pluripotent stem (hPS) cells. Here we present a completely defined, xeno-free medium that supports long-term propagation of hPS cells on uncoated tissue culture plastic. The medium consists of the Essential 8 (E8) formulation supplemented with inter- $\alpha$-inhibitor $(|\alpha|)$, a human serum-derived protein, recently demonstrated to activate key pluripotency pathways in mouse PS cells. I $\alpha$ l efficiently induces attachment and long-term growth of both embryonic and induced hPS cell lines when added as a soluble protein to the medium at seeding. | $\alpha$ l supplementation efficiently supports adaptation of feeder-dependent hPS cells to xeno-free conditions, clonal growth as well as single-cell survival in the absence of Rho-associated kinase inhibitor (ROCKi). This time-efficient and simplified culture method paves the way for large-scale, high-throughput hPS cell culture, and will be valuable for both basic research and commercial applications.

\footnotetext{
${ }^{1}$ Department of Medical Biochemistry and Microbiology, Box 582, Uppsala University, 75123 Uppsala, Sweden. ${ }^{2}$ Department of Immunology, Genetics and Pathology and Science for Life Laboratory, Box 815, Uppsala University, 75108 Uppsala, Sweden. ${ }^{3}$ Stem Cell Glycobiology Group, Wolfson Centre for Stem Cells, Tissue Engineering \& Modelling Room A59, University of Nottingham, NG7 2RD Nottingham, UK. ${ }^{4}$ GE Healthcare Bio-Sciences AB, Björkgatan 30, 75184 Uppsala, Sweden. Correspondence and requests for materials should be addressed to S.P.-G. (email: sara.pijuan@imbim.uu.se) or to C.A. (email: cecilia.anneren@ge.com).
} 
$\mathrm{H}$ uman pluripotent stem (hPS) cells, including human embryonic stem cells (hES cells) and induced pluripotent stem cells (hiPS cells), can self-renew indefinitely while retaining the capacity to differentiate into any somatic cell type. They therefore have great potential in various applications including basic developmental research, drug/toxicity screening and cell-based therapeutics ${ }^{1}$. The complex matrix requirements of hPS cells, which make up the hPS cell 'niche', are well documented and traditionally hPS cell expansion has necessitated culture on feeder cells and serum-containing media ${ }^{2}$. However, incompatibility of these complex, ill-defined conditions with pharmacological and medical applications has driven the development of alternative strategies combining defined media with improved surfaces. Solutions typically include surface immobilization of cell-binding motifs, such as integrin-binding proteins $s^{3,4}$, short peptides derived from vitronectin (VN), laminin $(\mathrm{LN})^{5,6}$, glycosaminoglycan (GAG)-binding peptides ${ }^{6,7}$ and synthetic polymers 8,9 . Current novel approaches use highthroughput combinatorial arrays to discover fully synthetic alternatives ${ }^{10}$. However, to date, these methods have not been widely implemented, being either too expensive or lacking the required reproducibility, leaving feeder cells or Matrigel ${ }^{11}$ in widespread use. Moreover, many hPS cell lines have also proven resistant to successful adaptation to feeder-free conditions. There are many hPS cell-specific issues that need to be addressed for optimal culture. Routine culture usually involves passage in small aggregates or clumps to avoid a loss of viability associated with dissociation (anoikis). The addition of ROCKi (Y-27632) to the hPS cell medium increases survival after single-cell passaging, but it is costly, particularly at scale ${ }^{12}$. Recent years have seen considerable efforts made to simplify and refine medium formulations, and the fully defined E8 medium is an important step forward in this regard ${ }^{5}$. Containing only eight components, all of them produced recombinantly, E8 is typically used together with a recombinant $\mathrm{VN}$ peptide which is preapplied to the tissue culture (TC) plastic as a coating for optimal cell attachment and survival (the one used in this study is Vitronectin-XF (VN-XF), distributed by Primorigen and StemCell Technologies). The combination of E8 and VN-XF has the benefits of being xeno-free and defined. However, as shown in the present study, this method does not robustly support cloning and single-cell passaging unless cells are pretreated for several hours with ROCKi. Large-scale and automated systems of the future will require affordable reagents available in large quantities, and simplified methodologies that can support efficient and reliable single-cell passaging.

The I $\alpha$ I protein family members are present at high concentrations $\left(0.6-1.2 \mathrm{mg} \mathrm{ml}^{-1}\right)$ in human serum ${ }^{13}$. Inter- $\alpha$ inhibitor (I $\alpha \mathrm{I})$, which is the most common family member, consists of two heavy chains ( $\mathrm{HCl}$ and $\mathrm{HC} 2$ ) and a bikunin (Bk) domain linked together by chondroitin sulphate ${ }^{13,14}$. The I $\alpha$ I complex has been associated to inflammation processes, hepatitis, cancer and even kidney diseases ${ }^{15-19}$. Traditionally, I $\alpha \mathrm{I}$ has been described as an extracellular matrix (ECM) component. The HCs are the only proteins known to covalently bind the ECM GAG hyaluronan (HA), while Bk when released from the HCs acts as a serine protease inhibitor ${ }^{14,20,21}$. However, recent reports demonstrate that the HC domains can additionally induce cell signalling $20,22-24$. Indeed, we recently showed that $\mathrm{I} \alpha \mathrm{I}$ (specifically its $\mathrm{HC} 2$ domain) activates the Yes/Yes-associated protein (YAP)/TEAD transcription factor pathway and induces expression of Oct4 and Nanog in mouse ES (mES) cells ${ }^{24}$.

In the present study we demonstrate, for the first time, that $\mathrm{I} \alpha \mathrm{I}$ and its HC2 domain promote attachment and survival of hPS cells. Moreover, we describe a new, simplified and time-efficient cell culture method based on the E8 medium supplemented with soluble I $\alpha \mathrm{I}$ (herein called E8:I $\alpha \mathrm{I}$ ). This method requires no surface coating and supports long-term propagation of hPS cells, clonal expansion and single-cell passaging even in the absence of ROCKi.

\section{Results}

I $\alpha$ I and its HC2 domain induce hPS cell attachment. To study whether I $\alpha$ I could support stem cell attachment, K2C hiPS cells were seeded onto standard TC-treated plastic in E8 medium supplemented with $50 \mu \mathrm{g} \mathrm{ml}^{-1} \mathrm{I} \alpha \mathrm{I}$. The purity of the isolated I $\alpha \mathrm{I}$ protein solution was thoroughly validated through silver staining and mass spectrometry analysis (Supplementary Fig. 1). Cell attachment was assessed $4 \mathrm{~h}$ after seeding using crystal violet staining, and $\mathrm{VN}-\mathrm{XF}$ coated plates $(\mathrm{E} 8 \mathrm{VNN})$ were used as positive control. Figure la shows how I $\alpha$ I supplementation induces similar cell attachment levels as VN-XF coating. The separate Bk and $\mathrm{HC} 1 \mathrm{I} \alpha \mathrm{I}$ domains were unable to support attachment when added in solution to the medium (Supplementary Fig. 2; Fig. 1a). However, when HC2 (but not HC1) was pre-coated onto standard TC-treated plastic, similar attachment levels, as with $\mathrm{I} \alpha \mathrm{I}$ supplementation, were achieved $4 \mathrm{~h}$ after seeding (Fig. 1a). Cells seeded in I $\alpha \mathrm{I}$ or on HC2-coated plates exhibited similar colony morphology and cell-cell contacts (defined by E-cadherin arrangement) as cells seeded on VN-XF (Fig. 1b; Supplementary Fig. 3). To further investigate the role of the different I $\alpha$ I domains in the hPS cell attachment we added antibodies against each of the three I $\alpha \mathrm{I}$ domains at the seeding step. Even though the use of the separate I $\alpha \mathrm{I}$ domains showed HC2 to be responsible for the hPS cell attachment, all three antibodies inhibited I $\alpha$ I-induced attachment regardless of which domain was being targeted, suggesting that antibody binding hinders I $\alpha$ I-mediated attachment when used in solution (Fig. 1c).

Serum albumin inhibits I $\alpha$ I-mediated attachment. When an alternative stem cell culture medium, mTeSR1 (which contains a high concentration of bovine serum albumin (BSA, 1\% w/w) and $\beta$-Mercaptoethanol, was used as basal medium for I $\alpha \mathrm{I}$, significantly lower cell attachment was achieved as compared with using E8 medium (determined by paired $t$-test). We therefore hypothesized that BSA may bind and sequester I $\alpha \mathrm{I}$, reducing its effective concentration in the medium. To test this, I $\alpha$ I-mediated attachment of the hES cell line HUES1 and the hiPS cell line $\mathrm{K} 2 \mathrm{C}$ was measured in $\mathrm{E} 8$ medium, E8 medium supplemented with $5 \% \mathrm{w} / \mathrm{v}$ BSA or mTeSR1 medium. Addition of BSA to the E8 medium significantly reduced I $\alpha$ I-mediated attachment (determined by paired $t$-test) to similar levels as those observed when using mTeSR1 (Fig. 1d). Albumin is commonly used to block non-specific binding to plastic or other surfaces because of its rapid and wide-ranging binding capacity. To confirm that the inhibition of I $\alpha$ I-mediated attachment observed in the presence of albumin was not due to a competition for surface coating, we pre-coated the surface with $50 \mu \mathrm{g} \mathrm{ml}^{-1}$ $\mathrm{I} \alpha \mathrm{I}$ for $1 \mathrm{~h}$ at room temperature (RT) before seeding hPS cells in either E8 medium or mTeSR1 medium. Again, the attachment was lower in mTeSR1 as compared with the E8 medium, further confirming that albumin can indeed reduce the ability of $I \alpha$ I to promote cell attachment, possibly by binding to it directly and thereby blocking its effect (Supplementary Fig. 2).

Integrins are involved in I $\alpha$ I-mediated attachment. The HCs contain a von Willebrand type A (vWA) domain, which has been reported to bind proteins such as VN via RGD (arginineglycine-aspartate) motifs ${ }^{25}$. To assess if the observed effect of I $\alpha$ I supplementation was mediated by integrin engagement of 

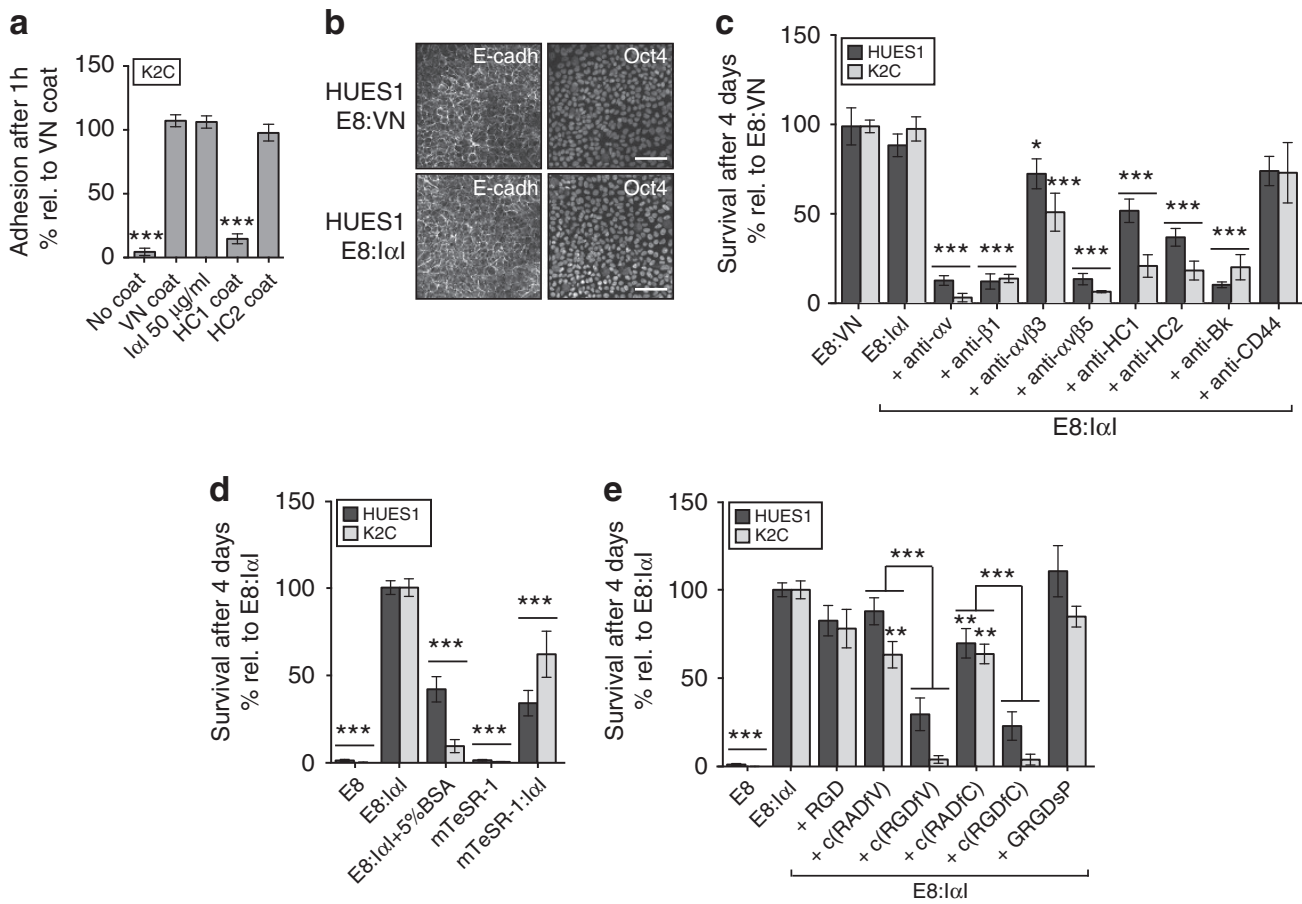

Figure 1 | I $\alpha \mathbf{l}$ and I $\mathbf{\alpha}$ l domain HC2 can support attachment and survival of hPS cells. (a) Quantification of cell attachment by crystal violet staining $4 \mathrm{~h}$ after seeding of hiPS cell line $\mathrm{K} 2 \mathrm{C}$ added to plates with $10 \mu \mathrm{g} \mathrm{ml}^{-1} \mathrm{VN}-\mathrm{XF}$ coating, $50 \mu \mathrm{g} \mathrm{ml}^{-1} \mathrm{| \alpha |}$ medium supplementation, $50 \mu \mathrm{g} \mathrm{ml}-1 \mathrm{HC} 1 \mathrm{coating}^{-1}$ $50 \mu \mathrm{g} \mathrm{ml}{ }^{-1} \mathrm{HC} 2$ coating. (b) Immunofluorescence of hES cell line HUES1 on VN-XF coating or lal supplement showing E-cadherin (left panel) and Oct4 (right panel), scale bar shows $100 \mu \mathrm{m}$. (c) Cell survival and growth assayed with crystal violet staining after 4 days of culture of HUES1 and K2C in E8 medium with $|\alpha|$ and different blocking antibodies, (d) E8 medium with or without $50 \mu \mathrm{g} \mathrm{ml}^{-1}|\alpha|(E 8:|\alpha|), 5 \%$ BSA (w/v) in E8: | $\alpha \mid$, and mTeSR1 medium with or without $|\alpha| ;(\mathbf{e})$ E8 medium supplemented with $|\alpha|$ and $|\alpha|$ together with different integrin-blocking peptides. All cell-number quantification experiments were performed in triplicate over three separate experiments. Bars show mean \pm s.e.m. and statistical analysis over the three independent experiments. Statistical analysis indicates significant differences with ${ }^{\star} P<0.05,{ }^{\star \star} P<0.001$ and ${ }^{\star \star \star}{ }^{\star} P<0.001$ determined by One-way ANOVA and Dunnett's post-test. Specific comparisons were done with paired t-test and are shown by a connective line between the two sets of samples.

RGD-motifs, a panel of RGD-blocking peptides were tested for their ability to inhibit I $\alpha$ I-mediated attachment (Fig. 1e). The linear RGD peptide H1830 did not have an effect, but the cyclic

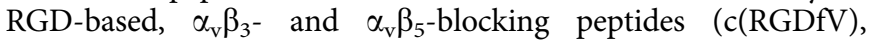
H-2574 (ref. 26)) and (c(RGDfC), H-7226 (ref. 27)) significantly reduced attachment as compared with their controls c(RADfV) and c(RADfC) (H-4088 and H-7232, respectively) (determined by paired $t$-test). These peptides block attachment to $\mathrm{VN}$ and $\mathrm{LN}$, which have been shown to support hPS cell attachment and survival ${ }^{4,5,7}$. The GRGDsP (H-3164)blocking peptide, which reduces attachment to fibronectin but does not interfere with VN-mediated binding, did not reduce $\mathrm{I} \alpha \mathrm{I}$-induced attachment (all peptides were from Bachem and are described in Supplementary Table 1).

To confirm the results obtained with the RGD-blocking peptides, we used blocking antibodies against VN and LNbinding integrins together with the E8:I $\alpha$ I method. The antibodies were added at the seeding step and cell survival and growth was assessed after 4 days. Blocking the $\alpha_{\mathrm{v}}$ and $\beta_{1}$ integrin domains and the $\alpha_{v} \beta_{5}$ integrin drastically affected attachment, while the $\alpha_{v} \beta_{3}$-blocking antibody reduced the attachment to a lesser extent (Fig. 1c). The $\beta_{1}$ integrin domain has been shown to be the main integrin domain responsible for hPS cell binding to $\mathrm{LN}^{3,4}$, while $\alpha_{\mathrm{v}}$ has been described as VN binding in hPS cells when present in $\alpha_{\mathrm{v}} \beta_{1}, \alpha_{\mathrm{v}} \beta_{3}$ and $\alpha_{\mathrm{v}} \beta_{5}$ complexes $^{28}$. Together, these results point towards the involvement of multiple integrin subunits in I $\alpha \mathrm{I}$-mediated attachment, in contrast to the limited number of subunits involved in binding to simple ECM-derived fragment coatings ${ }^{3}$.
HA is involved in I $\alpha$ I-mediated attachment. HA is a nonsulphated GAG found in many matrices throughout the body where it affects multiple functions including cell migration and proliferation. HA hydrogels have also proven useful for hPS cell culture and delivery ${ }^{29}$. The HCs of I $\alpha \mathrm{I}$ are the only proteins yet described to bind covalently to HA, and we therefore investigated if $\mathrm{HA}$ was involved in the I $\alpha \mathrm{I}$-mediated attachment of hPS cells. CD44 is a common HA-receptor and has been linked to cancer cell attachment and transformation following HA binding. However, we observed no inhibition of I $\alpha$ I-induced attachment in the presence of a CD44-blocking antibody (Fig. 1c). The involvement of GAGs in I $\alpha$ I-mediated attachment was further studied using a chemical inhibitor of GAG synthesis, 4-methylumbelliferone $(4-\mathrm{MU})^{30,31}$. The hES cell line HUES1 was treated with $0.6 \mathrm{mM}$ of 4 -MU for $24 \mathrm{~h}$ before passaging and seeded onto $\mathrm{VN}$-coated plates or E8:I $\alpha \mathrm{I}$, with $0.6 \mathrm{mM} 4-\mathrm{MU}$ supplementation. As shown in Supplementary Fig. 4, 4-MU treatment significantly reduced I $\alpha$ I-mediated attachment to $\sim 75 \%$ of the control samples, and this reduction was not observed for $\mathrm{VN}$-mediated attachment (determined by one-way ANOVA analysis and Dunnett's post-test against negative control E8:VN). Whilst it is noted that a reduction in GAG synthesis may impact multiple aspects of cell behaviour, attachment of 4-MU-treated cells could be fully recovered by adding exogenous high molecular weight HA (HMW-HA, 2,000-2,400 KDa) therefore suggesting that HA may be involved in E8:I $\alpha \mathrm{I}$-mediated attachment, however via a non-CD44-related mechanism (determined by one-way ANOVA analysis and Dunnett's post-test against negative control E8:VN, and paired $t$-test against 4-MU-treated E8:I $\alpha$ I sample). 
Live monitoring of the hES cell line HUES1 shows how $\mathrm{I} \alpha \mathrm{I}$ induces cell attachment and spreading over a similar timeframe and in a similar manner as the VN-XF coating (Supplementary Movie 1). Thus, I $\alpha$ I appears to exert a direct effect on cell attachment, similar to VN-XF coating, with attachment and spreading as concurrent events. The above data therefore point towards a complex attachment process, likely to involve several membrane receptors and extracellular ligands.

I $\alpha$ I efficiently supports single-cell passaging of hPS cells. To further evaluate I $\alpha$ I-induced hPS cell attachment and growth; HUES1 and K2C hPS cells were dissociated into small aggregates using a gentle enzyme-free reagent (Gentle Cell Dissociation Reagent (GCDR, Stem Cell Technologies)), or into single cells using TrypLE (a recombinant cell-dissociation enzyme, ThermoFisher) with ROCKi supplementation. The cells were then seeded using E8:VN or E8 with different I $\alpha$ I concentrations ranging from 0 to $500 \mu \mathrm{g} \mathrm{ml}^{-1}$. On day 4 , cell density was assessed using crystal violet staining. As shown in Fig. 2 and Supplementary Fig. $5,40-100 \mu \mathrm{g} \mathrm{ml}^{-1}$ of I $\alpha$ I was able to support attachment, characteristic colony morphology and positive alkaline phosphatase staining; $50 \mu \mathrm{g} \mathrm{ml}^{-1} \mathrm{I} \alpha \mathrm{I}$ was therefore selected for all subsequent experiments (and is denoted as E8:I $\alpha \mathrm{I}$ ). It was also confirmed that $\mathrm{I} \alpha \mathrm{I}$ addition is only required during seeding and the initial attachment process, and is not required in subsequent medium exchanges.

When sub-culturing in small aggregates, $50 \mu \mathrm{g} \mathrm{ml}^{-1}$ of $\mathrm{I} \alpha \mathrm{I}$ supplementation at the seeding step supported similar viability as $\mathrm{VN}-\mathrm{XF}$ coating. However, when performing single-cell splitting, almost no cells had survived after 4 days on VN-XF coating even with the addition of $10 \mu \mathrm{M}$ ROCKi to the culture during the first $24 \mathrm{~h}$ after seeding. In contrast, under these conditions, I $\alpha$ I supplementation supported very high cell survival, with cells reaching a monolayer after 4 days. Further analysis showed that $\mathrm{I} \alpha \mathrm{I}$ was also able to support survival and colony formation of dissociated single cells in the absence of ROCKi, although the survival rate was lower than with ROCKi (Fig. 3a,b). Live monitoring of hES cells (HUES1) cultured in the absence of ROCKi showed that although the cells initially attach and spread as single cells on VN-XF coating, they eventually lift off the plate and die, while HUES1 cells seeded in the presence of $I \alpha I$ exhibit decreased motility after seeding but stronger cell-to-cell contact formation, and grow as adherent colonies until they are ready to be passaged (Supplementary Movie 2).

As presented above, in the absence of ROCKi pre-treatment, no hPS cell survival was observed on VN-XF coating. A 1-h pre-treatment with ROCKi is usually recommended for single-cell splitting of hPS cells to increase cell survival ${ }^{12}$. However, in our hands, 1-h pre-treatment resulted in inconclusive results when using the E8:VN method, even at high seeding densities. To prevent this, the ROCKi pre-treatment step was increased to $5 \mathrm{~h}$. This longer pre-treatment improved the survival in E8:VN, but not to the level seen with E8:I $\alpha$ I. Thus, as shown in Supplementary Fig. 6a, there was a consistent higher cell density after 4 days in E8:I $\alpha$ I cultures when compared with VN-XF coating, from very low cell-seeding densities $\left(25\right.$ cells per $\left.\mathrm{cm}^{2}\right)$ up to typical seeding densities $\left(10^{3}\right.$ cells per $\left.\mathrm{cm}^{2}\right)$.

$I \alpha I$ protects from residual protease remaining after passage. $I \alpha I$ has been reported to act as protease inhibitor through its bikunin domain $^{32}$. E8 is a low-protein medium and therefore, small traces of protease remaining in the culture medium after splitting could result in cell damage and subsequent death due to the lack of protease inhibitors. TrypLE is a recombinant protease that has been reported to be more gentle on the cell surface, and is therefore recommended for stem cell culture ${ }^{33}$. To investigate if the reduced cell survival observed using $\mathrm{VN}$ coating together with single-cell splitting is caused by remaining traces of TrypLE in the medium, we used a 5-h ROCKi pre-treatment followed by TrypLE passaging with a dilution and centrifugation step to remove all TrypLE remains. The cells were then seeded with and without the addition of $1 \%(\mathrm{~V} / \mathrm{V})$ of TrypLE. Supplementary Fig. $6 \mathrm{~b}$ shows that while E8:I $\alpha$ I could support $\sim 100 \%$ survival of hES cell line HUES1 in 1\% TrypLE, VN coating was unable to support cell survival in $1 \%$ TrypLE. These data suggest that $\mathrm{I} \alpha \mathrm{I}$ is able to partially protect against residual TrypLE in the medium after passaging.

VN in solution does not support single-cell seeding. According to the manufacturer's recommendations, the $\mathrm{VN}$ peptide should be used as a coating agent in combination with E8 medium. To determine whether VN could be added in solution, similar to $\mathrm{I} \alpha \mathrm{I}$, cells were pre-treated with ROCKi for $5 \mathrm{~h}$ and single cells were then seeded on either $\mathrm{VN}$-coated plates or on uncoated plates in E8 medium supplemented with $10 \mu \mathrm{g} \mathrm{ml}^{-1} \mathrm{VN}$ or $50 \mu \mathrm{g} \mathrm{ml}^{-1} \mathrm{I} \alpha \mathrm{I}$. As shown in Supplementary Fig. $6 \mathrm{c}$, VN added in solution could not support attachment and survival to the same extent as $\mathrm{I} \alpha \mathrm{I}$ added in solution or coated $\mathrm{VN}$, demonstrating that $\mathrm{I} \alpha \mathrm{I}$-induced cell attachment is not only a result of rapid coating of the tissue culture plastic and that $\mathrm{VN}$ is only able to support attachment when used as a coating agent (Supplementary Fig. 6c).

I $\alpha$ I increases clonal survival of hPS cells. Live imaging of NCL1 hES cells seeded in E8:I $\alpha$ I with ROCKi after single-cell splitting revealed a colony forming from one single cell, suggesting that
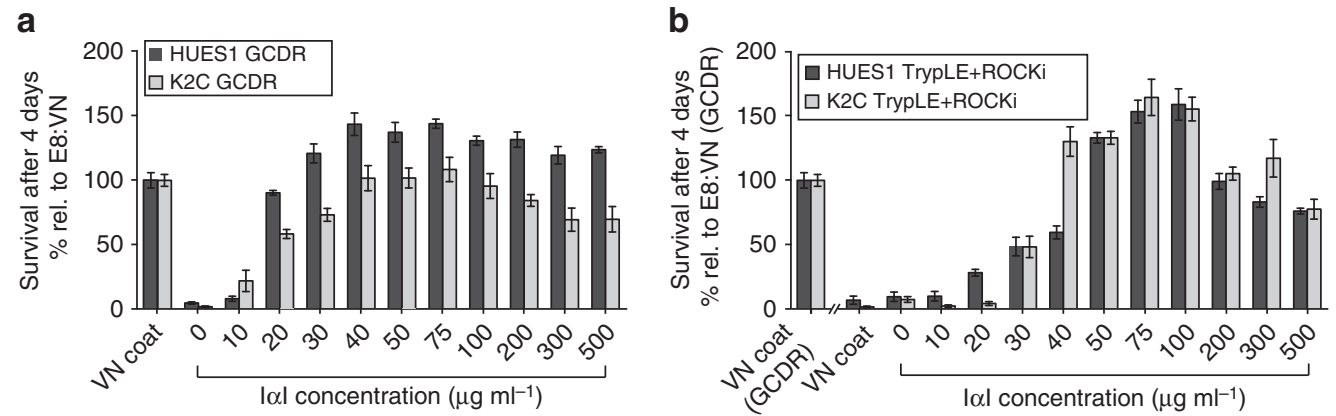

Figure 2 | lal attachment is concentration dependent. Dose-response curve of hPS cell lines HUES1 and K2C seeded as (a) small clumps (GCDR) or (b) single cells with ROCKi (TrypLE $+10 \mu \mathrm{M}$ ROCKi after seeding, no ROCKi pre-treatment) using increasing concentrations of I $\alpha$ l in E8 medium, compared with VN-XF coating. All cell-number quantification experiments were performed in triplicate over three separate experiments. Bars show mean \pm s.e.m. 
a

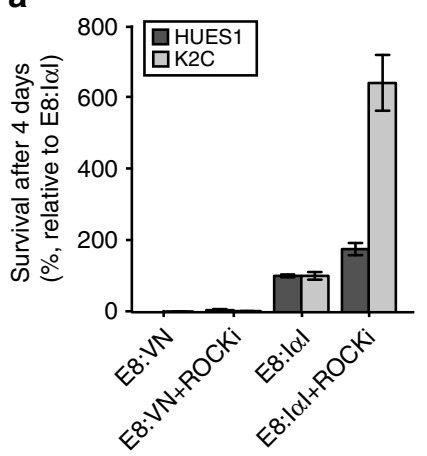

b

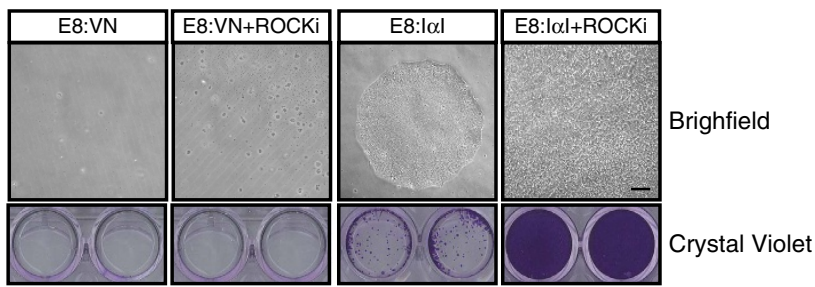

Figure 3 | lal increases survival after single-cell splitting. (a) Quantification of HUES1 and K2C cell survival and growth, using crystal violet staining, after 4 days in culture on VN-XF coat or with $|\alpha|$ supplementation after single-cell passaging with or without addition of $10 \mu \mathrm{M}$ ROCKi after seeding and no ROCKi pre-treatment (b) representative images for HUES1 cell line, brightfield pictures on the top and overview of crystal violet on the bottom. All cellnumber quantification experiments were performed in triplicate over three separate experiments. Bars show mean \pm s.e.m. in comparison to E8:I $\alpha$ l values.

Table 1 | I $\alpha$ I increases cloning efficiency of hPS cells.

\begin{tabular}{|c|c|c|c|c|}
\hline hPS line & Culture Method & Number of wells & Number wells with 1 colony & Cloning efficiency (\%) \\
\hline HUES1 & E8:VN & 298 & 7 & 2.3 \\
\hline HUES1 & $E 8:|\alpha|$ & 451 & 50 & 11.1 \\
\hline $\mathrm{K} 2 \mathrm{C}$ & E8:VN & 100 & 0 & 0 \\
\hline $\mathrm{H} 207$ & E8:VN & 100 & 1 & 1 \\
\hline $\mathrm{H} 207$ & $\mathrm{E} 8:|\alpha|$ & 93 & 7 & 7.5 \\
\hline $\mathrm{H} 181$ & E8:VN & 200 & 2 & 1 \\
\hline $\mathrm{K} 8 \mathrm{~F}$ & $E 8:|\alpha|$ & 284 & 32 & 11.3 \\
\hline NCL1 & $E 8:|\alpha|$ & 275 & 50 & 18.2 \\
\hline
\end{tabular}

Cloning efficiency of individualized HUES1, K2C, H207, H181, K8F and NCL1 hPS cells in 96-well plates using E8:I $\alpha$ ( E8 medium with $50 \mu g$ ml ${ }^{-1}$ I $\alpha$ l supplementation) or E8:VN (E8 medium on vitronectin-coated surface) with 5-h pre-treatment and supplementation of $10 \mu \mathrm{M}$ ROCKi for the first $48 \mathrm{~h}$. Assessment of colonies was done 14 days after seeding through MTT assay.

I $\alpha$ I can support clonal growth (Supplementary Movie 3). We therefore assessed the cloning success rate for six different hPS cell lines (K2C, HUES1, H207, H181, NCL1 and K8F) cultured in E8:I $\alpha$ I or E8:VN. High cloning rates of up to $18 \%$ were achieved with E8:I $\alpha$ I, and the cloning efficiency was consistently higher using the E8:I $\alpha$ I protocol as compared with E8:VN protocol in all five hPS cell lines tested, despite using a $5 \mathrm{~h}$ pre-treatment with ROCKi (Table 1).

Easy adaptation of hPS cell lines to E8:I $\alpha$ I. To ensure that the E8:I $\alpha$ I protocol can support robust propagation and pluripotency of different hPS cells, six hES cell lines (HUES1, H181, H207, OXF2, NCL1, huES3-Hb9::GFP) and two hiPS cell lines (K2C and $\mathrm{K} 8 \mathrm{~F}$ ), originally derived in four different laboratories, were adapted to E8:I $\alpha$ I. All hPS cell lines exhibited normal morphology in E8:I $\alpha$ I medium (Supplementary Fig. 3a). The E8:I $\alpha$ I protocol showed better adaptation efficiency of the hES cell line OXF2 from feeder-cell culture as compared with E8:VN. Through a simple step-wise adaptation protocol, combining E8:I $\alpha \mathrm{I}$ and single-cell passaging using TrypLE dissociation and ROCKi (Fig. 4a), we achieved $100 \%$ adaptation efficiency of five different feeder-dependent hPS cell lines (OXF2, NCL1, huES3::Hb9rGFP, $\mathrm{K} 2 \mathrm{C}$ and $\mathrm{K} 8 \mathrm{~F}$ ), with approximately four-fold better yield as compared with the E8:VN protocol for OXF2 hES cells (Fig. 4b). Healthy colony formation was achieved at passage 1 and negligible differentiation was observed from passage 2 (Fig. 4c).
Successful adaptation of NCL1 was particularly noteworthy since this line had, in our hands, proved incompatible with feeder-free culture. These data suggest enhanced adaptation efficiency of feeder-dependent hPS cell lines to E8:I $\alpha$ I. Assessment after 5-10 passages proved expected stem cell marker expression (Fig. 4c).

I $\alpha$ I supports long-term propagation of hPS cells. Three hES cell lines (HUES1, H207 and H181) and one hiPS cell line (K2C) were then cultured for 15 or more passages in E8:I $\alpha \mathrm{I}$. The E8:VN protocol was used as positive control and, for adequate comparison, cells were split in small clumps for both protocols. All cell lines grew as tight colonies with negligible signs of differentiation regardless of culture condition. E8:I $\alpha \mathrm{I}$ also supported freeze-thaw cycles with survival rates after recovery similar to E8:VN. Proliferation rate was assessed for up to 40 days (10 passages) and showed similar growth rates independent of culture protocol and cell line (Fig. 5a). Immunofluorescence staining highlighted strong and specific expression of stem cell markers in all cell lines after 15 or more passages in both protocols (Fig. 6a; Supplementary Fig. 7).

The Human Stem Cell Pluripotency TaqMan array (ThermoFisher) was performed to characterize expression profiles of the four hPS cell lines after long-term propagation focusing on marker genes for pluripotency, stemness and differentiation ${ }^{34}$. No significant differences in transcription profiles were found between culture conditions (as determined 
a

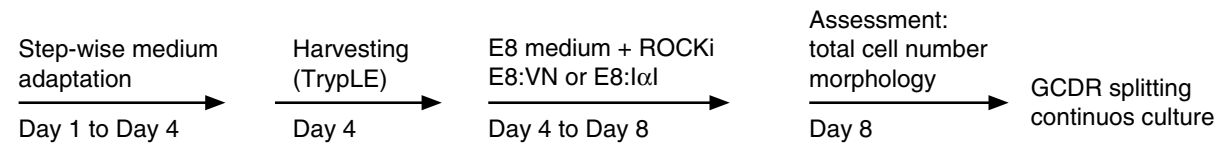

b

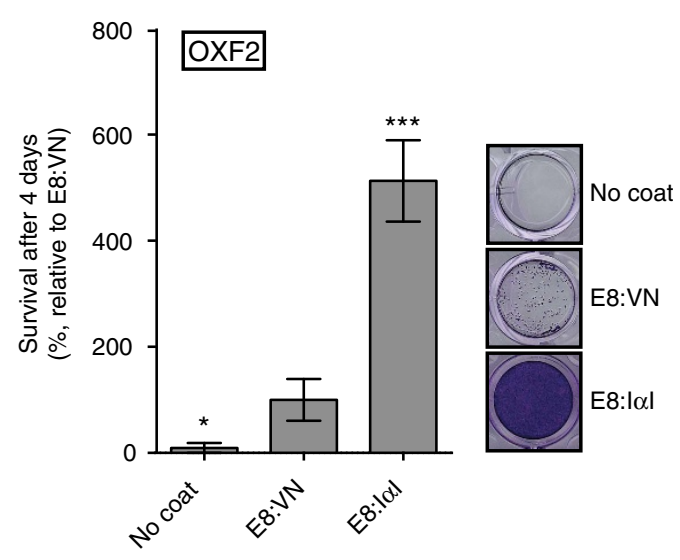

C Passage 1 Passage 2 Passage 3

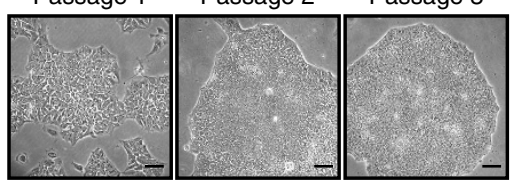

OFX2 passage 5 in $\mathrm{E} 8:|\alpha|$

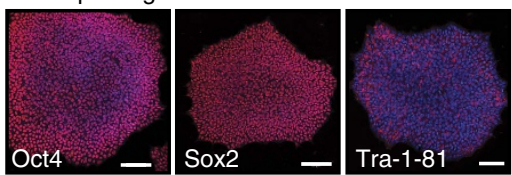

NCL1 passage 10 in E8:I $\alpha$ l

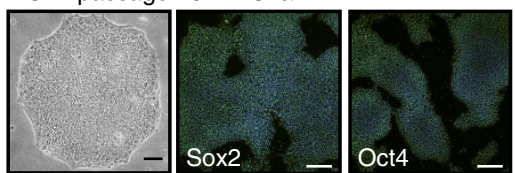

Figure 4 | I $\mathbf{\alpha}$ l achieves high-adaptation efficiency of feeder-dependent hPS cell lines. (a) Step-wise adaptation sequence for feeder-dependent hPS cell lines to E8:I $\alpha$ l and E8:VN (b) Crystal violet staining and quantification of OXF2 cell survival and growth 4 days after adaptation to E8:I $\alpha$ l or E8:VN, right panel shows representative images. (c) Colony morphology and immunofluorescence staining of stem cell factors Oct4, Nanog and Tra-1-81 of OXF2 after adaptation of hES cell lines OXF2 and NCL1 from feeders and serum-containing medium to E8:I lal. Scale bars show 100 $\mu$ m. All cell-number quantification experiments were performed in triplicate over three separate experiments. Bars show mean \pm s.e.m. and statistical analysis shows significant difference with ${ }^{\star} P<0.05,{ }^{\star \star} P<0.001$ and ${ }^{\star \star \star} P<0.001$ (determined by One-way ANOVA and Dunnett's post-test) in comparison to E8:VN values.

by multiple comparisons using two-way ANOVA and Sidak's Multiple test), indicating that I $\alpha$ I does not induce differentiation or reduce expression of stem cell related markers (Fig. 5b,c; Supplementary Fig. 8; Supplementary Data 1). In fact, when relative expression values for stem cell markers were assessed closely, it was found that hPS cells grown in E8:I $\alpha$ I showed a more compact expression profile than hPS cells grown in E8:VN (Fig. 5b,c; Supplementary Fig. 8). The cells were then subjected to spontaneous differentiation by embryoid body formation and subsequent plating onto Matrigel with serum-containing medium. After 4 weeks, all hPS cell lines, independent of previous culture condition, showed successful differentiation into the three germ layers as assessed by immunofluorescence staining for specific markers (Fig. 6a; Supplementary Fig. 9a,b). TaqMan array was then performed on the differentiated hPS cell samples and again, no differences in transcriptional profiles were found between the two culture methods (Fig. 5b; Supplementary Data 1). Moreover, as shown in Supplementary Movie 4, K2C hiPS cells successfully produced beating cardiomyocytes after 40 passages in E8:I $\alpha \mathrm{I}$.

I $\alpha$ I supports directed endoderm differentiation. Endoderm has been the most difficult germ layer to generate in vitro ${ }^{35}$. To investigate if $\mathrm{I} \alpha \mathrm{I}$ could also support directed differentiation protocols for hPS cells, we combined the STEMDiff Definitive Endoderm Kit (TeSR-E8 optimized, StemCell Technologies) with I $\alpha$ I. Briefly, four hPS cell lines grown in E8:I $\alpha$ I for 10 or more passages were pre-conditioned to endoderm before being split as single cells and seeded using I $\alpha$ I. All four hPS cell lines attached normally and survived throughout the five-day differentiation protocol. The results showed that cells cultured in E8:I $\alpha$ I can be readily induced to differentiate into endoderm, generating a monolayer of endoderm precursor cells, positive for the key early endoderm markers Sox7, Sox17 and HNF3 $\beta$ (ref. 36; Fig. 6b; Supplementary Fig. 10).

I $\alpha$ I does not increase genetic abnormalities. To ensure that $I \alpha I$ does not increase the level of mutations or chromosomal abnormalities after long-term culture when compared with current methods, all hPS cell lines were subjected to G-banding and the Human OmniExpressExome array (Illumina, SNP\&SEQ Technology Platform, Uppsala University). Single-nucleotide polymorphism (SNP) array data were analysed both by total number of copy-number $(\mathrm{CN})$ calls, and number of shared $\mathrm{CN}$ calls between early and later passage (Supplementary Tables 3 and 4; Supplementary Fig. 11). G-banding analysis showed few but equal amounts of abnormalities in both culture methods (Supplementary Table 2; Supplementary Fig. 12). Further analysis done by using SNP-array data indicates that the $\mathrm{CN}$ profiles changed during culturing regardless of protocol used and cell line examined and that the two examined protocols perform equally well in retaining genetic profiles (Supplementary Table 3). A stricter $\mathrm{CN}$ analysis was also performed by comparing the number of shared $\mathrm{CN}$ calls between early and late passages in each of the four cell lines under the two protocols and for all 123 $\mathrm{CN}$ calls (Supplementary Table 4). Overall, we found no difference in the number of genetic alterations detected (Wilcoxon rank-sum-test; $W=10.5, P=0.8082$ ). No increase in genetic abnormalities was observed in E8:I $\alpha \mathrm{I}$ as compared with E8:VN and the starting passages, suggesting that $\mathrm{I} \alpha \mathrm{I}$ does not increase the risk of genetic aberrations or selection for advantageous mutations in long-term culture. Even though the presence of abnormalities in all conditions may seem alarming, the hPS cell lines used in this study were obtained at high passages, and it has been reported that hPS cell lines can develop abnormalities after long-term culture in vitro ${ }^{37}$. Indeed, a 
a

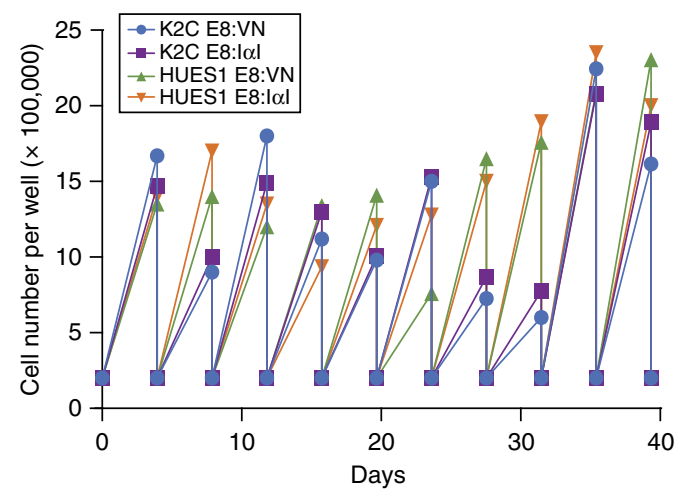

b

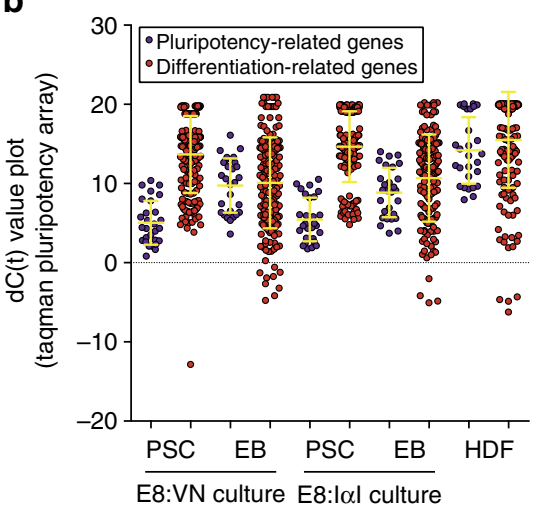

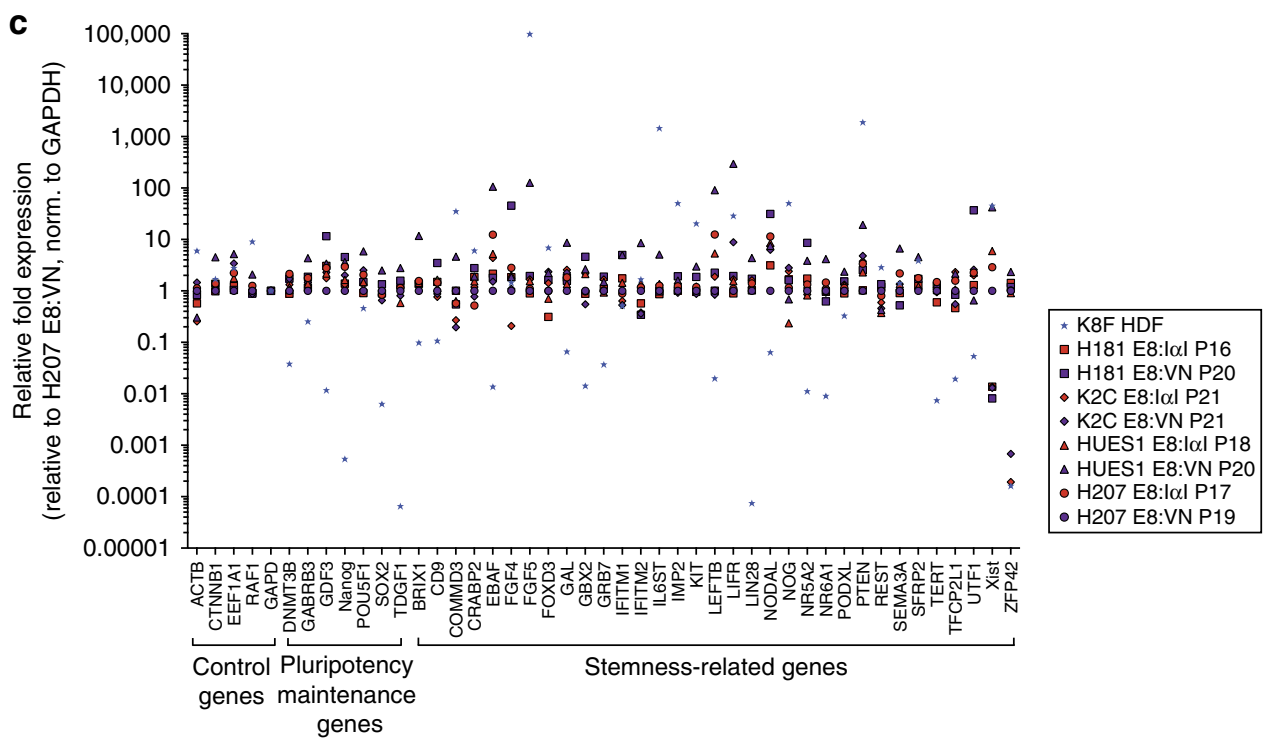

Figure 5 | Ial maintains pluripotency and self-renewal of hPS cells in long-term culture. (a) Growth curve of K2C hiPS cell line and HUES1 hES cell line in either E8:VN or E8:Ial for 10 passages. (b) Dot-plot illustrating combined $\mathrm{dC}(\mathrm{t}$ ) values of pluripotency genes (purple dots) and differentiation genes (red dots) obtained using the Taqman pluripotency array on hPS cell lines HUES1, H207, H181 and K2C after 16 or more passages in E8:VN or E8:I $\alpha$ I (PSC) and after differentiation through EB formation (EB) compared with human dermal fibroblasts (HDF), note that higher $C(t)$ values translate to lower expression, and average distribution is represented with yellow bars. (c) Relative expression values for 45 housekeeping, pluripotency and stemness genes across the four hPS cell lines after long-term passaging in E8:VN (outlined blue symbols) or E8:I $\alpha$ ( (outlined red symbols), relative expression to H207 grown in E8:VN and normalized to GAPDH expression, and compared to human dermal fibroblast line K8F (HDF, blue stars). No statistical difference was found (determined by Two-way ANOVA and Sidak's post-test). Note that E8:Ial samples exhibit less variation in pluripotency gene expression levels as compared to the E8:VN samples.

previous large-scale analysis of 125 independent hPS cell lines from 38 different laboratories reported that small variations similar to the ones present in this study could be found in all samples, and documented that these are consistent with previous structural variants studies made on human populations, therefore dependent on their source genome and not from their in vitro culture $^{38}$.

\section{Discussion}

The last few years have seen a marked refinement of hPS cell culture methods, enabling the use of better defined culture conditions as well as simplified handling protocols, essential for the widespread use of hPS cells. The potential of hPS cells as renewable cell sources for drug toxicity screening, human cell therapy and regenerative medicine has rendered traditional cell culture methods, based on ill-defined components, unsuitable ${ }^{39}$. For this purpose, several defined surfaces have been developed to improve hPS cell derivation and expansion ${ }^{40,41}$. However, none of the currently available methods fulfil all requirements for simple, robust, cost-efficient and safe production of hPS cells. The combination of E8 medium with VN-XF ${ }^{5}$ coating is both defined and animal-component free, and has been proven to successfully support long-term culture of hPS cells when passaged as small clumps. However, this method requires a surface pre-coating step and, as shown in the present work, is inefficient when hPS cells are passaged as single cells.

In this paper we present $I \alpha I$ as a protein able to induce attachment of hPS cells onto plastic without the need for a precoating step. This is the first time that $I \alpha I$ has been reported to induce attachment in in vitro culture, particularly remarkable considering the extensive and numerous efforts to identify substrates for hPS cell culture. During this study we discovered that high concentrations of albumin in culture medium reduce I $\alpha$ I-induced attachment to $\sim 50 \%$. These results suggest that albumin binds $I \alpha I$ and reduces its ability to induce cell attachment, both when present as a soluble factor and a pre-coated agent. This is possibly the reason why this is the 
a

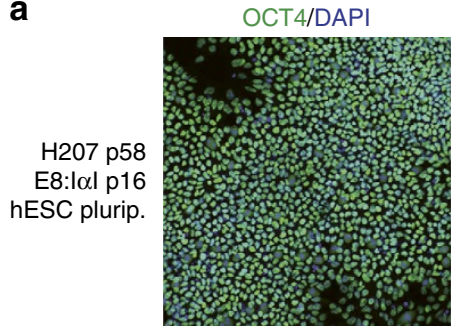

aSMA/DAPI

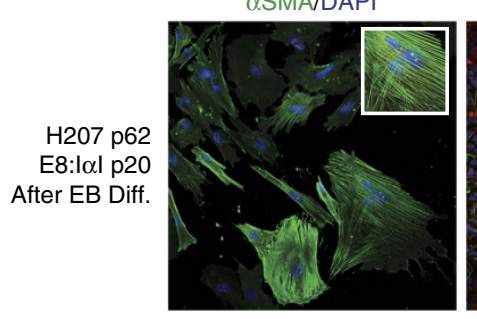

b

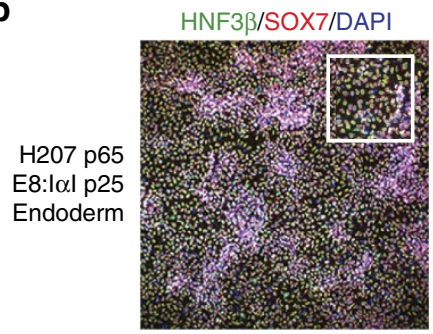

SOX2/DAPI

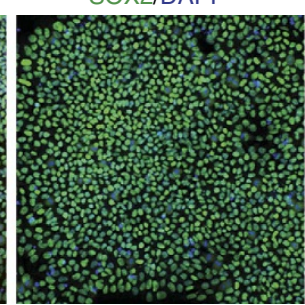

B3Tubulin/Nestin/DAPI

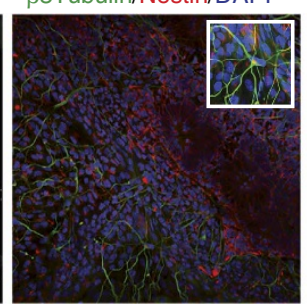

SOX17/DAPI

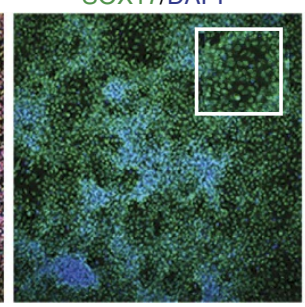

NANOG/DAPI

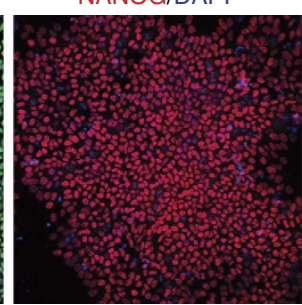

AFP/PDGF-R/DAPI

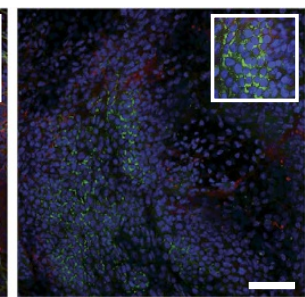

Oct4/NANOG/DAPI

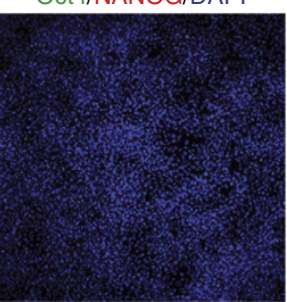

SSEA4/DAPI
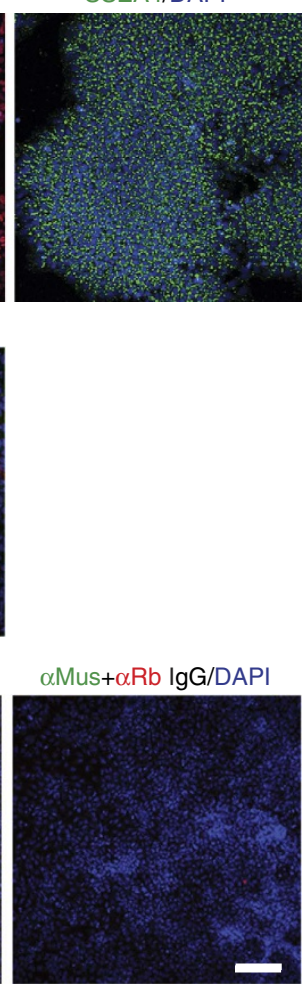

Figure 6 | lal maintains pluripotency and differentiation capacity in long-term culture. Immunofluorescence staining of $\mathrm{H} 207 \mathrm{hES}$ cell line for (a) stem cell markers Oct4 (green), Sox2 (green), Nanog (red) and SSEA-4 (green) after 16 passages in E8:Ial, and for the three germ layers after 4 weeks of differentiation through embryoid body (EB) formation: mesoderm with alpha smooth muscle actin (SMA, green), ectoderm with Nestin (red) and $\beta$-IIItubulin (green) and endoderm with alpha-fetoprotein (AFP, green) and PDGF-receptor (red), and (b) after endoderm directed differentiation for endoderm markers Sox7 (red), Sox17 (green) and HNF3ß (green) and stem cell markers Oct4 (green) and Nanog (red), as well as negative control with secondary antibodies. All samples were co-stained with DAPI (Blue) for nuclei detection. Scale bars show $100 \mu \mathrm{m}$, white boxes show close-up images.

first study linking $\mathrm{I} \alpha \mathrm{I}$ to cell attachment, as the majority of traditional cell culture media contain high concentrations of albumin.

$\mathrm{I} \alpha \mathrm{I}$ is a relatively large protein that consists of three distinct globular domains: Bk, HC1 and HC2. We found that antibodies targeted against any of the I $\alpha$ I domains could block I $\alpha$ I-induced attachment of hPS cells when I $\alpha$ I was used in solution. However, the only domain that could support hPS cell growth on its own was HC2, and only when HC2 was used as a coating agent. These results concur with our previous report showing that the HC2 domain of I $\alpha$ I, but not the HC1 domain, can activate pluripotency-related transcription in $\mathrm{mES} \mathrm{cells}^{24}$. It has also been reported that transfer of the $\mathrm{HC}$ domains to $\mathrm{HA}$ requires the $\mathrm{HC} 2$ domain to be present, as part of the $\mathrm{I} \alpha \mathrm{I}$ molecule or as a separate domain (for example, HC2-Bk) ${ }^{20,23}$, confirming the distinct biological activity of the HCs. While $\mathrm{HC} 1$ and $\mathrm{HC} 2$ share functional domains (a vault protein inter- $\alpha$-trypsin (VIT) domain and a vWA domain), outside of these regions there is considerable heterogeneity, and overall, human $\mathrm{HC} 1$ and $\mathrm{HC} 2$ only share $39 \%$ amino acid identity.

While little is known about the function of the VIT domain, the vWA domain has been reported to bind many proteins via RGD domains, including $\mathrm{VN}^{25}$. Thus, to assess if the I $\alpha \mathrm{I}$-induced attachment was integrin mediated, we used RGD-blocking peptides as well as integrin-blocking antibodies and found that multiple integrin subunits were likely to be involved in the process, pointing to a more complex interaction than the traditional integrin-binding protein attachment of $\mathrm{VN}$ - and LN-based coatings. Since the HCs of I $\alpha \mathrm{I}$ are the only proteins reported to bind covalently to $\mathrm{HA}$, we also investigated the potential role for HA in I $\alpha \mathrm{I}$-mediated attachment. Blocking the HA receptor CD44 did not have any effect on I $\alpha$ I-induced attachment. However, reducing GAG synthesis using an inhibitor decreased I $\alpha \mathrm{I}$-mediated attachment, indicating that HA may play a role I $\alpha \mathrm{I}$-mediated attachment. Overall, our results point to I $\alpha$ I-induced attachment being the result of a synergistic combination of several extracellular molecules and surface receptors. Indeed, $\mathrm{I} \alpha \mathrm{I}$ has been reported to interact with several extracellular molecules, including $\mathrm{HA}^{20}$, integrin-binding proteins such as $\mathrm{VN}^{25}$, and the complement system ${ }^{42}$. In vivo, many different processes have been linked to $\mathrm{I} \alpha \mathrm{I}$, including the cumulus formation of the oocyte $^{43}$, fibrosis and angiogenesis ${ }^{22}$, tissue repair ${ }^{25}$, amniotic membrane formation ${ }^{44,45}$, arthritis ${ }^{46}$ and others. I $\alpha$ I is proving to be a very versatile protein with a wide variety of functions, which most likely depend on the different partners it can interact with in each situation. Clearly, further study will be needed to completely unravel the mechanism underpinning I $\alpha \mathrm{I}$-mediated attachment.

In this study we present the first hPS cell culture protocol that overcomes the need for specialized surface coating or functionalization, while still being defined and animal-component free. This method is highly time efficient, as coating is a timeconsuming step in hPS cell culture methods. We demonstrate that E8:I $\alpha$ I improves single-cell passaging and cloning efficiency, without the need for a lengthy ROCKi pre-treatment, and can protect from residual protease, thus making automated handling simpler and enabling cell-number control and high-throughput experimental design. Long-term propagation and characterization of four different hPS cell lines and successful adaptation of a total of eight different hPS cells lines prove that this culture method is 
reliable and robust. Moreover, we have shown that $\mathrm{I} \alpha \mathrm{I}$ can support a directed differentiation protocol. E8:I $\alpha$ I can also support freeze-thaw cycles in the same manner as commercially available methods. The I $\alpha \mathrm{I}$ used in this study was derived from a side fraction of human plasma used for commercial factor IX production, with purity above $95 \%$, as described previously ${ }^{14,21,24}$. I $\alpha$ I has a long shelf-life and can potentially be purified and prepared in large quantities from human plasma at GMP grade and at a reasonable cost. Future development will also investigate on the possibility of using recombinant $\mathrm{I} \alpha \mathrm{I}$, or parts thereof. In conclusion, this simple method offers a reliable and cost-efficient solution to the current need for defined hPS cell culture conditions and has great potential to transform research, pharmacological and clinical application of hPS cells and their derivatives.

\section{Methods}

Materials and cell lines. Cells: hES cell lines H181, H207 (kindly provided by Dr Hovatta from Karolinska Institutet, Stockhom), HUES1 and HUES3-Hb9::GFP (kindly provided Dr Melton and Dr, Eggan from Harvard Stem Cell Institute and Dr. Koslova from Uppsala University), OXF2 and NCL1 (UK Stem Cell Bank), and hiPS cell lines $\mathrm{K} 2 \mathrm{C}$ and $\mathrm{K} 8 \mathrm{~F}$ (generated by Dr Schuster, Uppsala University). Primary antibodies: mouse anti-Oct4 (POUF5F1) cl. 7F9.2 (Millipore), rabbit anti-Nanog (ab109250, Abcam), mouse anti-Sox2 (ab171380, Abcam), mouse anti-SSEA-4 (MAB4304, Millipore), mouse anti-Tra-1-81 (MAB4381, Millipore), mouse anti-Tra-1-60 (MAB4360, Millipore), mouse anti- $\alpha$-Fetoprotein (AFP) receptor (MAB4086, Millipore), rabbit anti-PDGF $\beta$ receptor (sc-432, Santa Cruz Biotechnology), mouse anti-Neuron specific $\beta$-III-tubulin (MAB1195, R\&D), rabbit anti-E-cadherin (\#3195, Cell Signaling Technology), mouse anti- $\alpha$-Smooth Muscle Actin ( $\alpha$ SMA) (CBL171, Millipore), rabbit anti-nestin (ab105389, Abcam), mouse anti-HNF3 $\beta$ (sc-101060, Santa Cruz Biotechnology), mouse anti-Sox17 (SAB3300046, Sigma), rabbit anti-Sox7 (HPA009065, Sigma). Blocking antibodies used were: mAB13 (ref. 47) anti- $\beta 1$ (kindly provided by Dr. Kenneth Yamada, NIDCR, USA), anti- $\alpha$ v L230 (ab94704, Abcam), anti- $\alpha$ v $\beta 3$ (MAB1976, Millipore), anti- $\alpha$ v 35 (ab24694, Abcam), anti-ITIH1 K-16 (sc-33944, Santa Cruz Biotechnology), anti-ITIH2 N-17 (sc-21974, Santa Cruz Biotechnology), anti-Bk P-20 (sc-21597, Santa Cruz Biotechnology), rat anti-CD44 KM114 (kindly provided by Dr. Paraskevi Heldin, Uppsala University). Secondary antibodies: Alexa Fluor 488 goat anti-mouse (ThermoFisher), Alexa Fluor 555 goat anti-rabbit (ThermoFisher). Other reagents: Hoechst 33342 (ThermoFisher), Matrigel ${ }^{\mathrm{TM}}$ Matrix hES-qualified (Corning), RGD-based blocking peptides (all from Bachem, described in Supplementary Table 1), Y-27632 (ROCK inhibitor, ROCKi, StemCell Technologies), 25\% hES-qualified BSA (Invitrogen), high molecular weight HA 2,000-2,400 kDa (HMW-HA, Cat No. 73641, Sigma-Aldrich), and 4-Methylumbelliferone (4-MU, M1381, Sigma).

Cell culture. The hPS cell starting cultures were on Matrigel coating (Corning) in mTeSR1 medium (StemCell Technologies) ${ }^{11}$ or on feeder cells (gamma-irradiated MEFs or CRL-2429 HFFs) in $20 \%$ KOSR and $8 \mathrm{ng} \mathrm{ml}^{-1}$ bFGF supplemented KO-DMEM ${ }^{48}$. Continuous culture on VN-XF and E8 medium was performed following manufacturer's instructions. Briefly, vitronectin coating was performed by diluting VN-XF (StemCell Technologies \#07180) in Dilution Buffer (StemCell Technologies \#07183) to a final concentration of $10 \mu \mathrm{g} \mathrm{ml}^{-1}$, then immediately using the solution to coat non-tissue culture-treated cultureware, gently mixing the solution by rocking. The solution was allowed to coat for at least $1 \mathrm{~h}$ at RT or overnight $(\mathrm{ON})$ at $4{ }^{\circ} \mathrm{C}$. The coated well was washed once with the dilution buffer before use. The hPS cells were harversted using the Gentle Cell Dissociation Reagent (GCDR, StemCell Technologies \#07174), the E8 medium was removed and the appropriate volume of GCDR was added and incubated for 5-8 min at RT. Then the solution was removed and the appropriate volume of E8 medium added, the colonies were scrapped using a cell lifter (Corning, CLS3008) and broken down by pipetting up and down $4-5$ times. After that the cells were seeded using a dilution according to the initial density, typically ranging from 1:4 to 1:10

Culture of hPS cells in E8:Ial. For the continuous culture of hPS cells in E8:I $\alpha$ I the cells were grown until appropriate size before splitting using GCDR (StemCell Technologies \#07174). Briefly, the medium was removed; the appropriate volume of GCDR was added to the wells and incubated at RT for 5-8 min. The GCDR solution was removed and the appropriate volume of E8 medium was added, the colonies were then harvested using a cell lifter (Corning, 900020) and broken down by pipetting up and down 4-5 times. The wells were split 1:4 to 1:10 (depending on starting cell density), fresh E8 medium together with the hPS cells was added into a new, uncoated, tissue culture-treated plastic (Corning). Finally, $50 \mu \mathrm{g} \mathrm{ml}^{-1} \mathrm{I} \alpha \mathrm{I}$ was added into the well and mixed by gentle rocking. The culture medium was changed daily using standard E8 medium. I $\alpha$ I supplementation was not necessary after the initial seeding step.
Adaptation of feeder-dependent PS cells to E8:Ial. The hPS cells cultured on feeder cells were adapted to E8 medium using a 4-day step-wise adaptation from the original medium to the E8 medium. On day 4, the cells were harvested using TrypLE and then seeded in E8 medium supplemented with $10 \mu \mathrm{M}$ Y-27632 onto tissue culture-treated plastic with $50 \mu \mathrm{g} \mathrm{ml}{ }^{-1} \mathrm{I} \alpha \mathrm{I}$ supplementation or onto VN-XF coated surfaces, cell density used was approximately $5 \times 10^{3}$ cell per $\mathrm{cm}^{2}$. The medium was then changed daily until day 4 , when cells were passaged or assessed for total cell number. After the first passage, continuous culture passaging was done using GCDR.

Growth-rate assessment. The hPS cell lines were split for routine culture using a GCDR and collected using a cell lifter. For the growth-rate assessment, duplicates were kept for every condition. One well was passaged with GCDR for continued culture, while the other was passaged with TrypLE for cell-number measurement with a TCP counter (Bio-Rad).

Cryopreservation of hPS cells. The cells were harvested using GCDR and a cell lifter. The freezing medium used was mFreezer or FreSR-S (StemCell Technologies) and the final cell concentration ranged from $4-10 \times 10^{6}$ cells per ml. Cell solution $(1 \mathrm{ml})$ was used per cryovial, the cells were then transferred to a $-80^{\circ} \mathrm{C}$ freezer for a slow-cooling using an isopropanol freezing container. After $24 \mathrm{~h}$, the cryovials were transferred to permanent storage in vapour phase liquid nitrogen.

Differentiation. Embryoid body (EB) formation was performed using the aggrewell system (StemCell Technologies), harvesting the cells using TrypLE with 1-h ROCKi pre-treatment and seeding an average of 2,000 cells per EB and with ROCKi addition. The EBs were then kept in GMEM 20\% FBS and suspension for 2 weeks and then plated onto Matrigel (Corning) or Synthemax (vitronectin, Corning) for 2 more weeks. Beating cardiomyocytes areas were filmed after 3-4 weeks of growth using a light-phase microscope coupled to a NikonE990 camera.

Purification of human Ial. A side fraction from the commercial manufacturing of factor IX (Pharmacia-Upjohn, Stockholm, Sweden) was fractionated using gel filtration chromatography on a HiPrep 26/60 Sephacryl S-400 HR column using the ÄKTA automated chromatography system (both from GE Healthcare). This process generates more than $95 \%$ pure I $\alpha$ I. For the release of the heavy chains, a solution of $1 \mathrm{mg} \mathrm{ml}^{-1} \mathrm{I} \alpha \mathrm{I}$ in PBS was subjected to $0.05 \mathrm{M} \mathrm{NaOH}$ treatment for $15 \mathrm{~min}$ at RT and then dialyzed against $20 \mathrm{mM}$ sodium phosphate $\mathrm{pH} 7.6$ and subsequently applied to an anion-exchange chromatography for separation using the ÄKTA system (MonoQ 5/50 GL; GE Healthcare) ${ }^{49}$. Fractions were analysed in $8 \%$ acrylamide SDS-PAGE gels and Coomassie Brilliant Blue staining. Unless specified otherwise, protein concentrations were determined by UV measurements and using the absorbance coefficients for the protein moieties of I $\alpha \mathrm{I}, \mathrm{HC} 1$ and $\mathrm{HC} 2$ obtained from Bloom et al..$^{14}$ The protein solutions were concentrated and stored at $-20^{\circ} \mathrm{C}$.

Crystal violet attachment assay. The hPS cells were collected with either with GCDR or TrypLE. Approximately $5 \times 10^{3}$ cell per $\mathrm{cm}^{2}$ were seeded, unless specified otherwise. For single-cell splitting, $4-5 \mathrm{~h}$ of $10 \mu \mathrm{M}$ ROCKi pre-treatment was used (unless stated otherwise) and the dissociation reagent was TrypLE (ThermoFisher). For single-cell splitting $10 \mu \mathrm{M}$ ROCKi was also added for the first $24 \mathrm{~h}$ after seeding, unless specified otherwise ${ }^{12}$. After the specified time for attachment and/or growth, total cell number was assessed ${ }^{50}$. Briefly, the plates were washed twice with PBS to remove non-attached cells and fixed using ice-cold methanol for $10 \mathrm{~min}$, and subsequently stained with $0.4 \% \mathrm{w} / \mathrm{v}$ crystal violet in methanol for $5 \mathrm{~min}$. The crystal violet was then thoroughly washed away using distilled water, and the plates were dried before adding $175 \mu \mathrm{l}$ of $0.1 \%$ SDS solution per 24 -well plate well. After $1-2 \mathrm{~h}$ on a tilting table at RT, quantification was performed measuring optical density at $570 \mathrm{~nm}$ with Multiscan PLUS (Labsystems). All cell-number quantification experiments were performed in triplicates, with three separate independent experiments performed, unless specified otherwise. The samples were also periodically randomized so as to ensure no position-related variations.

Attachment blocking. The hPS cells were harvested as small clumps using GCDR and seeded at $5 \times 10^{3}$ cells per $\mathrm{cm}^{2}$ in E8 supplemented with $50 \mu \mathrm{g} \mathrm{ml}^{-1} \mathrm{I} \alpha \mathrm{I}$ (E8:I II) with the addition of each antibody at $5 \mu \mathrm{g} \mathrm{ml}{ }^{-1}$ for the integrin-blocking antibodies, and $20 \mu \mathrm{g} \mathrm{ml}^{-1}$ for the I $\alpha \mathrm{I}$-binding antibodies for the first $24 \mathrm{~h}$. The RGD-blocking peptides were also added at the seeding step at a concentration of $25 \mu \mathrm{M}\left(9-15 \mu \mathrm{g} \mathrm{ml}^{-1}\right)$. The cells were cultured for 4 days before cell survival and growth was assessed using crystal violet staining.

GAG synthesis inhibition. The inhibitor 4-Methylumbelliferone (4-MU) was used to inhibit GAG synthesis in the hPS cells to assess its effect of on the I $\alpha$ I-mediated attachment. Briefly, hPS cells were treated for $24 \mathrm{~h}$ in E8 medium supplemented with $0.6 \mathrm{mM} 4-\mathrm{MU}$ or with $6 \mu \mathrm{l} \mathrm{MeOH}$ as a negative control. This concentration has been reported to decrease HA synthesis without inducing apoptosis in cancer cell lines ${ }^{31}$. After treatment the cells were harvested as small clumps using GCDR and seeded onto VN coating or with I $\alpha$ I, with the addition of $\mathrm{MeOH}$ or 
4-MU for the first $24 \mathrm{~h}$ after seeding. Moreover, in the 4-MU-treated samples $100 \mu \mathrm{g} \mathrm{ml}^{-1} \mathrm{HMW}-\mathrm{HA}$ was also added to assess the effect of exogenous HA.

Directed endoderm differentiation. Directed endoderm differentiation was performed using the STEMDiff Definitive Endoderm Kit (TeSR-E8 optimized, StemCell Technologies), according to the manufacturer's instructions. Four hPS cell lines cultured on E8:I $\alpha$ I conditions were grown for 4 days. At day four, the medium was changed to Pre-differentiated supplemented E8 medium and used for daily exchanges for $48 \mathrm{~h}$. The cells were then washed with PBS and split using GCDR and thorough pipetting to achieve a single-cell suspension. The single suspension was centrifuged and resuspended in E8 medium supplemented with I $\alpha \mathrm{I}$ and pre-differentiation reagent, and seeded at $2 \times 10^{5}$ cells per $\mathrm{cm}^{2}$. After $24 \mathrm{~h}$, the medium was changed to StemDiff Definitive Endoderm Basal medium supplemented with reagents A and B. On day 2, the medium was changed into StemDiff Definitive Endoderm Basal medium supplemented with reagent B, and this medium was used to exchange daily until day 5 . The cells were then fixed and used for immunocytochemistry analysis.

Immunocytochemistry. Cells were fixed with cold $4 \%$ paraformaldehyde (Sigma) for $15 \mathrm{~min}$ and then blocked with $0.5 \% \mathrm{BSA}$ and $0.3 \%$ Triton X-100 in PBS (all from Sigma). The primary antibodies anti-Oct4 (1:300), anti-Nanog (1:100), anti-Sox2 (1:100), anti-SSEA-4 (1:200), anti-Tra-1-60 (1:100) and anti-Tra-1-81 (1:100), anti- $\beta$ III-Tubulin (1:100), anti-Nestin (1:200), anti- $\alpha$ SMA (1:200), anti-AFP (1:50), anti-PDGF $\beta$ receptor (1:50), anti-E-cadherin (1:100), anti-Sox7 (1:50), anti-Sox17 (1:50), anti-HNF3 $\beta$ (1:100), were added to the cells and incubated in a humid chamber at $4{ }^{\circ} \mathrm{C}$ overnight, rinsed with PBS, and subsequently incubated with Alexa Fluor 555 anti-rabbit (1:500) and/or Alexa Fluor 488 anti-mouse (1:500) for $60 \mathrm{~min}$ at room temperature. After rinsing with PBS and co-staining with Hoechst 33342, coverslips were mounted using fluoromount (Sigma), and analysed with inverted confocal fluorescent microscopy (LSM700, Zeiss). All images were uniformly processed using Adobe Photoshop CS6 (Version 13.0.6 x64).

Human stem cell pluripotency taqman array. Total RNA was extracted using an RNeasy Mini kit (Qiagen) following manufacturer's instructions. An RNA nano6000 chip and a Bioanalyser2100 (Agilent) were used to assay RNA quality, and only samples with an RNA integrity number (RIN) $\geq 8$ were selected for the array. cDNA was prepared from $1 \mu \mathrm{g}$ of total RNA using iScript (Bio-Rad) according to manufacturer's instructions, and then mixed with TaqMan Gene Expression Master Mix and loaded onto a TaqMan Human Stem Cell Pluripotency Low Density Array (TLDA, ThermoFisher). The arrays were run on a 7900HT Real-Time PCR System (Applied Biosystems). The data were processed with RQ Manager 1.2.1 software and relative expression $(\mathrm{dC}(\mathrm{t}))$ was calculated using GAPDH as a control and used to compare across all cell lines using the statistical programme GraphPad Prism 6. The hierarchical cluster analysis was performed by Genesis $^{51}$. All arrays were run at the SciLifeLab Genome facility (Uppsala, Sweden).

G-banding karyotype. Cultures of up to $70-80 \%$ confluency were treated with $100 \mathrm{ng} \mathrm{ml}^{-1}$ colcemide (KaryoMax colcemide Solution $\left(10 \mu \mathrm{g} \mathrm{ml}^{-1}\right)$, Gibco) overnight, and harvested using TrypLE (ThermoFisher). The cells were subsequently treated with freshly prepared hypotonic solution $(75 \mathrm{mM} \mathrm{KCl}$, Sigma) for $30 \mathrm{~min}$ at $37^{\circ} \mathrm{C}$ followed by a triple fixator treatment (3:1 Methanol:Acetic acid, both by Sigma) for $20 \mathrm{~min}$ each. The dropping of fixed cell suspension on cold wet microscope slides was used to achieve metaphases, which were subsequently processed using Trypsin (Sigma) and stained with Giemsa staining solution (Sigma) for G-banding analysis ${ }^{52}$. For each sample, twenty metaphases were analysed with IKAROS-software (MetaSystems). Description and specification of chromosomal aberrations was performed following guidelines from ISCN 2013.

SNP array and genotyping. SNP genotyping with Illumina HumanOmniExpressExome-array version 8v1-2_A, encompassing $>964.000$ single-nucleotide polymorphism (SNP)-markers, was performed by the SNP\&SEQ technology Platform in Uppsala (www.genotyping.se), according to the manufacturer's instructions. Each of the four hPS cell lines (that is, H181, H207, HUES1 and K2C) were analysed in three separate SNP-array experiments. Total DNA was extracted from cell culture growth at $60-70 \%$ confluence using the DNeasy Blood \& Tissue Kit (Cat. No. 69504, Qiagen). DNA concentration and purity was determined by ultraviolet measurement. A first analysis was performed after 2-5 passages of growth for initial reference, and subsequently, two analyses were performed per cell line after 16-21 passages in E8:VN or E8:I $\alpha \mathrm{I}$ culture. The $\mathrm{CN}$ calls of the autosomal chromosomes were illustrated using the Nexus software.

Copy-number analysis. Illumina output files were analysed using NexusCopy-Number version 7.5 (BioDiscovery, CA, USA). Linear systematic correction and the 'SNP-FASST2 segmentation' algorithm for CN-calling with default calling parameters was applied. A first $\mathrm{CN}$ analysis was performed on the autosomal chromosomes for each cell line after $2-5$ passages. Strict $\mathrm{CN}$-calling criteria (100\% overlap of $\mathrm{CN}$ calls and $P<0.05$ in Nexus software) were applied. After this, each of the four cell lines was grown to 16-21 passages, applying either the E8:I $\alpha \mathrm{I}$ or the E8:VN protocol. Subsequently, new $\mathrm{CN}$-analyses using the same settings in Nexus software was performed and the results were compared to the $\mathrm{CN}$ calls from earlier. The number of shared CN calls between the early and later passages in each of the four cell lines was used to quantify changes introduced during culturing within each cell line under the E8:I $\alpha \mathrm{I}$ and E8:VN protocols, respectively. We used Wilcoxon rank-sum-test in the statistical software R v3 to test for differences in the observed number of shared $\mathrm{CN}$ calls from the E8:I $\alpha \mathrm{I}$ and E8:VN protocols.

Clonal assay and MTT Tetrazolium assessment. The starting hPS cells were pre-treated for $4-5 \mathrm{~h}$ with $10 \mu \mathrm{M}$ Y-27632 before harvesting them using TrypLE (ThermoFisher). Briefly, $1 \mathrm{ml}$ TrypLE was added to a pre-treated 6-well plate well of hPS cells ready to passage. The cells were incubated with TrypLE for $8 \mathrm{~min}$ before removal and addition of $1 \mathrm{ml} \mathrm{E} 8$ medium with $10 \mu \mathrm{M}$ ROCKi, the cells were harvested with a cell lifter. Cell clumps were broken down by pipetting and the solution was passed through a $40 \mu \mathrm{m}$ pore cell strainer to ensure single-cell suspension (StemCell Technologies). The cells were then counted using the TCP counter (Bio-Rad). After that the cells were diluted down to 100 cells in $10 \mathrm{ml}$ of E8 medium with $10 \mu \mathrm{M}$ ROCKi, mixed and seeded on VN coating or with I $\alpha \mathrm{I}$ supplementation in $100 \mu \mathrm{l}$ per each of 100 wells (50 wells per 96-well plate) so as to achieve approximately one cell per well. After two weeks the survival and formation of colonies was assayed visually and through MTT assay. Briefly, MTT (3-(4,5-dimethylthiazol-2-yl)-2,5-diphenyltetrazolium bromide) tetrazolium was dissolved in $\mathrm{E} 8$ medium at a final concentration of $0.5 \mathrm{mg} \mathrm{ml}^{-1}$, the solution was filtered and added to the wells from the clonal assay and incubated at $37^{\circ} \mathrm{C}$ and $5 \%$ $\mathrm{CO}_{2}$ for $3 \mathrm{~h}$. After confirming the staining of the existing colonies, the MTT solution was removed and $20 \%$ SDS was added. The plates were incubated $\mathrm{O} / \mathrm{N}$ and the absorbance was read at 570 and $630 \mathrm{~nm}$. This is a measure for viable cells. Wells with more than one colony were removed from the final result summary.

Mass spectrometry analysis. Two samples of $\mathrm{I} \alpha \mathrm{I}$ working solution (purified separately) were mixed with $\beta$-mercaptoethanol and run through a $12 \%$ acrylamide SDS-PAGE denaturating gel. Band development was done through silver staining as described previously ${ }^{53}$. The samples were in-gel digested with trypsin according to a standard protocol and analysed by Liquid Chromatography Mass Spectrometry (LC-Orbitrap MS/MS) at the MS Facility, SciLifeLab, Uppsala University. The proteins were reduced, alkylated and in-gel digested by trypsin according to a standard operating procedure. Thereafter the samples were dried and resolved in $15 \mu \mathrm{l} 0.1 \%$ FA (formic acid). The peptides were separated in reversed-phase on a C18-column and electrosprayed on-line to an LTQ Velos Orbitrap mass spectrometer (Thermo Finnigan). Tandem mass spectrometry was performed applying CID. The database searches were performed using the Sequest algorithm, embedded in Proteome Discoverer 2.1 (ThermoFisher), against human proteins in the Swissprot database (2015). The search criteria for protein identification were set to at least two matching peptides of $95 \%$ confidence level per protein.

Live imaging of hPS cell growth. Human PS cell lines were seeded onto 6-well plates either as small clumps with GCDR or as single cells with TrypLE and $10 \mu \mathrm{M}$ Y-27632 (no pre-treatment). The imaging was performed with the oCelloscope 04C5 (Unisensor), placed inside a standard normoxic incubator. Pictures were taken from the seeding point and then every $15 \mathrm{~min}$ for up to 4 or 5 days (specified for each video file). The imaging was performed using the Uniexplorer 3.2.0.3621 CRL3 software (Unisensor). The exported avi files were further edited using the VideoPad Video Editor (Freeware)

Statistical analysis. Experiments were performed in at least three independent experiments and in triplicate for each experiment. The data is presented as mean \pm s.e.m. For the crystal violet cell analysis data multiple statistical analysis was done using one-way ANOVA with Dunnett's post-test, when applicable and stated in the figure legends. Paired $t$-test was used to compare two specific samples within groups, and the result is shown over a line connecting the two samples within the graph. The Human Stem Cell Pluripotency Taqman Array data was analysed using Two-way ANOVA and Sidak's Multiple comparison's test. All these statistical analyses were done using GraphPad Prism version 5.00d for Mac (GraphPad Software), and the data presented shows ${ }^{\star} P<0.05,{ }^{* *} P<0.001$ and ${ }^{* * *} P<0.001$ unless otherwise specified. For the copy-number analysis a Wilcoxon rank-sum-test in the statistical software $\mathrm{R} v 3$ was used.

Data availability. Data supporting the findings of this study are available within the article and its Supplementary Information files and from the corresponding author upon reasonable request. The SNP-array genotyping data have been deposited in the NCBI-based Gene Expression Omnibus (GEO) database (http://www.ncbi.nlm.nih.gov/geo/) under the accession code GSE82103. 


\section{References}

1. Carpenter, M. K., Frey-Vasconcells, J. \& Rao, M. S. Developing safe therapies from human pluripotent stem cells. Nat. Biotechnol. 27, 606-613 (2009).

2. Stojkovic, M. et al. Derivation of human embryonic stem cells from day-8 blastocysts recovered after three-step in vitro culture. Stem Cells 22, 790-797 (2004).

3. Rodin, S. et al. Clonal culturing of human embryonic stem cells on laminin-521/E-cadherin matrix in defined and xeno-free environment. Nat. Commun. 5, 3195 (2014)

4. Rodin, S. et al. Long-term self-renewal of human pluripotent stem cells on human recombinant laminin-511. Nat. Biotechnol. 28, 611-615 (2010).

5. Chen, G. et al. Chemically defined conditions for human iPSC derivation and culture. Nat. Methods 8, 424-429 (2011).

6. Klim, J. R., Li, L., Wrighton, P. J., Piekarczyk, M. S. \& Kiessling, L. L. A defined glycosaminoglycan-binding substratum for human pluripotent stem cells. Nat. Methods 7, 989-994 (2010).

7. Melkoumian, Z. et al. Synthetic peptide-acrylate surfaces for long-term self-renewal and cardiomyocyte differentiation of human embryonic stem cells. Nat. Biotechnol. 28, 606-610 (2010).

8. Villa-Diaz, L. G. et al. Synthetic polymer coatings for long-term growth of human embryonic stem cells. Nat. Biotechnol. 28, 581-583 (2010).

9. Brafman, D. A. et al. Long-term human pluripotent stem cell self-renewal on synthetic polymer surfaces. Biomaterials 31, 9135-9144 (2010).

10. Mei, Y. et al. Combinatorial development of biomaterials for clonal growth of human pluripotent stem cells. Nat. Mater. 9, 768-778 (2010).

11. Ludwig, T. E. et al. Feeder-independent culture of human embryonic stem cells. Nat. Methods 3, 637-646 (2006).

12. Watanabe, K. et al. A ROCK inhibitor permits survival of dissociated human embryonic stem cells. Nat. Biotechnol. 25, 681-686 (2007).

13. Josic, D. et al. Proteomic characterization of inter-alpha inhibitor proteins from human plasma. Proteomics 6, 2874-2885 (2006).

14. Blom, A. M., Morgelin, M., Oyen, M., Jarvet, J. \& Fries, E. Structural characterization of inter-alpha-inhibitor. Evidence for an extended shape. J. Biol. Chem. 274, 298-304 (1999).

15. Atmani, F., Glenton, P. A. \& Khan, S. R. Role of inter-alpha-inhibitor and its related proteins in experimentally induced calcium oxalate urolithiasis. Localization of proteins and expression of bikunin gene in the rat kidney. Urol. Res. 27, 63-67 (1999).

16. van den Broek, I. et al. The absolute quantification of eight inter-alpha-trypsin inhibitor heavy chain 4 (ITIH4)-derived peptides in serum from breast cancer patients. Proteomics Clin. Appl. 4, 931-939 (2010).

17. Jayapalan, J. J., Ng, K. L., Shuib, A. S., Razack, A. H. \& Hashim, O. H. Urine of patients with early prostate cancer contains lower levels of light chain fragments of inter-alpha-trypsin inhibitor and saposin B but increased expression of an inter-alpha-trypsin inhibitor heavy chain 4 fragment. Electrophoresis 34, 1663-1669 (2013).

18. Sira, M. M., Behairy, B. E., Abd-Elaziz, A. M., Abd Elnaby, S. A. \& Eltahan, E. E. Serum inter-alpha-trypsin inhibitor heavy chain 4 (ITIH4) in children with chronic hepatitis C: relation to liver fibrosis and viremia. Hepat. Res. Treat. 2014, 307942 (2014).

19. Hamm, A. et al. Frequent expression loss of Inter-alpha-trypsin inhibitor heavy chain (ITIH) genes in multiple human solid tumors: a systematic expression analysis. BMC Cancer 8, 25 (2008).

20. Sanggaard, K. W. et al. The transfer of heavy chains from bikunin proteins to hyaluronan requires both TSG-6 and HC2. J. Biol. Chem. 283, 18530-18537 (2008).

21. Hess, K. A., Chen, L. \& Larsen, W. J. Inter-alpha-inhibitor binding to hyaluronan in the cumulus extracellular matrix is required for optimal ovulation and development of mouse oocytes. Biol. Reprod. 61, 436-443 (1999).

22. Garantziotis, S. et al. Serum inter-alpha-trypsin inhibitor and matrix hyaluronan promote angiogenesis in fibrotic lung injury. Am. J. Respir. Crit. Care. Med. 178, 939-947 (2008).

23. Werbowetski-Ogilvie, T. E. et al. Isolation of a natural inhibitor of human malignant glial cell invasion: inter alpha-trypsin inhibitor heavy chain 2. Cancer Res. 66, 1464-1472 (2006).

24. Pijuan-Galito, S., Tamm, C. \& Anneren, C. Serum Inter-alpha-inhibitor activates the Yes tyrosine kinase and YAP/TEAD transcriptional complex in mouse embryonic stem cells. J. Biol. Chem. 289, 33492-33502 (2014).

25. Adair, J. E. et al. Inter-alpha-trypsin inhibitor promotes bronchial epithelial repair after injury through vitronectin binding. J. Biol. Chem. 284, 16922-16930 (2009)

26. Legler, D. F., Wiedle, G., Ross, F. P. \& Imhof, B. A. Superactivation of integrin alphavbeta3 by low antagonist concentrations. J. Cell. Sci. 114, 1545-1553 (2001).

27. Yang, X. et al. cRGD-functionalized, DOX-conjugated, and (6)(4)Cu-labeled superparamagnetic iron oxide nanoparticles for targeted anticancer drug delivery and PET/MR imaging. Biomaterials 32, 4151-4160 (2011).
28. Gil, J. E. et al. Vitronectin promotes oligodendrocyte differentiation during neurogenesis of human embryonic stem cells. FEBS Lett. 583, 561-567 (2009).

29. Prestwich, G. D. Hyaluronic acid-based clinical biomaterials derived for cell and molecule delivery in regenerative medicine. J. Control Release 155, 193-199 (2011).

30. Hiraga, T., Ito, S. \& Nakamura, H. Cancer stem-like cell marker CD44 promotes bone metastases by enhancing tumorigenicity, cell motility, and hyaluronan production. Cancer Res. 73, 4112-4122 (2013).

31. Lokeshwar, V. B. et al. Antitumor activity of hyaluronic acid synthesis inhibitor 4-methylumbelliferone in prostate cancer cells. Cancer Res. 70, 2613-2623 (2010).

32. Potempa, J., Kwon, K., Chawla, R. \& Travis, J. Inter-alpha-trypsin inhibitor. Inhibition spectrum of native and derived forms. J. Biol. Chem. 264, 15109-15114 (1989).

33. Ellerstrom, C., Strehl, R., Noaksson, K., Hyllner, J. \& Semb, H. Facilitated expansion of human embryonic stem cells by single-cell enzymatic dissociation. Stem Cells 25, 1690-1696 (2007).

34. International Stem Cell, I. et al. Characterization of human embryonic stem cell lines by the International Stem Cell Initiative. Nat. Biotechnol. 25, 803-816 (2007).

35. Wells, J. M. \& Melton, D. A. Vertebrate endoderm development. Annu. Rev. Cell. Dev. Biol. 15, 393-410 (1999).

36. Seguin, C. A., Draper, J. S., Nagy, A. \& Rossant, J. Establishment of endoderm progenitors by SOX transcription factor expression in human embryonic stem cells. Cell Stem Cell 3, 182-195 (2008).

37. Nguyen, H. T., Geens, M. \& Spits, C. Genetic and epigenetic instability in human pluripotent stem cells. Hum. Reprod. Update 19, 187-205 (2013).

38. International Stem Cell, I. et al. Screening ethnically diverse human embryonic stem cells identifies a chromosome 20 minimal amplicon conferring growth advantage. Nat. Biotechnol. 29, 1132-1144 (2011).

39. Martin, M. J., Muotri, A., Gage, F. \& Varki, A. Human embryonic stem cells express an immunogenic nonhuman sialic acid. Nat. Med. 11, 228-232 (2005)

40. Celiz, A. D. et al. Materials for stem cell factories of the future. Nat. Mater. 13, 570-579 (2014).

41. Higuchi, A. et al. Design of polymeric materials for culturing human pluripotent stem cells: Progress toward feeder-free and xeno-free culturing. Prog. Polym. Sci. 39, 1348-1374 (2014).

42. Okroj, M. et al. Heavy chains of inter alpha inhibitor (IalphaI) inhibit the human complement system at early stages of the cascade. J. Biol. Chem. 287, 20100-20110 (2012).

43. Carrette, O., Nemade, R. V., Day, A. J., Brickner, A. \& Larsen, W. J. TSG-6 is concentrated in the extracellular matrix of mouse cumulus oocyte complexes through hyaluronan and inter-alpha-inhibitor binding. Biol. Reprod. 65, 301-308 (2001).

44. Zhang, S., He, H., Day, A. J. \& Tseng, S. C. Constitutive expression of interalpha-inhibitor (IalphaI) family proteins and tumor necrosis factor-stimulated gene-6 (TSG-6) by human amniotic membrane epithelial and stromal cells supporting formation of the heavy chain-hyaluronan (HC-HA) complex. J. Biol. Chem. 287, 12433-12444 (2012).

45. Shay, E., He, H., Sakurai, S. \& Tseng, S. C. Inhibition of angiogenesis by HC.HA, a complex of hyaluronan and the heavy chain of inter-alpha-inhibitor, purified from human amniotic membrane. Invest. Ophthalmol. Vis. Sci. 52, 2669-2678 (2011).

46. Wisniewski, H. G., Burgess, W. H., Oppenheim, J. D. \& Vilcek, J. TSG-6, an arthritis-associated hyaluronan binding protein, forms a stable complex with the serum protein inter-alpha-inhibitor. Biochemistry 33, 7423-7429 (1994).

47. Mould, A. P., Akiyama, S. K. \& Humphries, M. J. The inhibitory anti-betal integrin monoclonal antibody 13 recognizes an epitope that is attenuated by ligand occupancy. Evidence for allosteric inhibition of integrin function. J. Biol. Chem. 271, 20365-20374 (1996)

48. Hovatta, O. et al. A culture system using human foreskin fibroblasts as feeder cells allows production of human embryonic stem cells. Hum. Reprod. 18, 1404-1409 (2003).

49. Enghild, J. J., Thogersen, I. B., Pizzo, S. V. \& Salvesen, G. Analysis of interalpha-trypsin inhibitor and a novel trypsin inhibitor, pre-alpha-trypsin inhibitor, from human plasma. Polypeptide chain stoichiometry and assembly by glycan. J. Biol. Chem. 264, 15975-15981 (1989).

50. Miyazaki, T. et al. Laminin E8 fragments support efficient adhesion and expansion of dissociated human pluripotent stem cells. Nat. Commun. 3, 1236 (2012).

51. Sturn, A., Quackenbush, J. \& Trajanoski, Z. Genesis: cluster analysis of microarray data. Bioinformatics 18, 207-208 (2002).

52. Meisner, L. F. \& Johnson, J. A. Protocols for cytogenetic studies of human embryonic stem cells. Methods 45, 133-141 (2008).

53. Shevchenko, A., Wilm, M., Vorm, O. \& Mann, M. Mass spectrometric sequencing of proteins silver-stained polyacrylamide gels. Anal. Chem. 68, 850-858 (1996). 


\section{Acknowledgements}

The following individuals and institutions provided reagents and cell lines: Dr E. Fries from Uppsala University (Purified human I $\alpha$ I, HCs and fraction of human serum), I.M. Lööf from Pharmacia-Upjohn (side fraction from the commercial production of factor IX), Dr E. Kopper from the University of Manchester and Dr K. Yamada from the National Institute of Health (L230 and MAb13 antibodies), Dr O. Houvatta from Karolinska Institutet (H181 and H207), Dr D. Melton and Dr K. Eggan from Harvard Stem Cell Institute and Dr E. Kozlova from Uppsala University (HUES1, Hb9::GFP), and the UK Stem Cell Bank (OXF2, NCL1). This work was supported by the Swedish Research Council, Jaenssons' Foundations and Faculty of Medicine of Uppsala University and an International Research Fellowship from the Leverhulme Trust. This work was supported by the Science for Life Laboratory Mass Spectrometry Based Proteomics Facility in Uppsala. Genotyping was performed by the SNP\&SEQ technology Platform in Uppsala (www.genotyping.se). The facility is part of the National Genomics Infrastructure supported by the Swedish Research Council for Infrastructures and Science for Life Laboratory, Sweden. The SNP\&SEQ Technology Platform is also supported by the Knut and Alice Wallenberg Foundation.

\section{Author contributions}

S.P-G.: Conception and design, provision of study material, collection and assembly of data, data analysis and interpretation, manuscript writing, final approval of manuscript. C.T.: conception and design, provision of study material, final approval of manuscript. J.S.: provision of study material, collection and/or assembly of data, data analysis and interpretation, final approval of manuscript. M.S.: collection and/or assembly of data data analysis and interpretation, final approval of manuscript. L.F.: Data analysis and interpretation, final approval of manuscript. C.L.R.M.: Provision of study material, manuscript writing, final approval of manuscript. C.A.: Conception and design, financial support, administrative support, manuscript writing, final approval of manuscript.

\section{Additional information}

Supplementary Information accompanies this paper at http://www.nature.com/ naturecommunications

Competing interests statement: C.A. works for GE Healthcare Bio-Sciences AB that has submitted the following patent applications covering these findings: Title: Method for cell culture. Application number/year: US14780873A/2015 and EP2014775556A/2014. Priority Number/year: SE201350400A/2013 and WO2014SE50373A/2014. S.P.G, C.T. and C.A. are co-inventors on the patent. The remaining authors declare no competing financial interests.

Reprints and permission information is available online at http://npg.nature.com/ reprintsandpermissions/

How to cite this article: Pijuan-Galitó, S. et al. Human serum-derived protein removes the need for coating in defined human pluripotent stem cell culture. Nat. Commun. 7:12170 doi: $10.1038 /$ ncomms12170 (2016)

\section{(c) (i)}

This work is licensed under a Creative Commons Attribution 4.0 International License. The images or other third party material in this article are included in the article's Creative Commons license, unless indicated otherwise in the credit line; if the material is not included under the Creative Commons license, users will need to obtain permission from the license holder to reproduce the material. To view a copy of this license, visit http://creativecommons.org/licenses/by/4.0/

(C) The Author(s) 2016 